\title{
Comparative Performance Analysis of AODV Parameter for ZigBee Network using Artificial Neural Network
}

\author{
Prativa P. Saraswala \\ Research Scholar \\ V.T Patel Dept. of Electronics and \\ Communication \\ Charusat, Changa
}

\author{
Jaymin Bhalani, $\mathrm{PhD}$ \\ Associate professor \\ Dept of Electronics and \\ Communication, \\ Babaria Institute of Technology, \\ Vadodara
}

\author{
Sandhya Sharma \\ Assistant Professor \\ Dept of Electrical Engg, Faculty of \\ Technology and Engg, \\ The Maharaja Sayajirao University \\ of Baroda
}

\begin{abstract}
This paper emphasizes on the signal transmission range of Zigbee network based on IEEE 802.15.4 standard using Simulink-based simulator called TRUE TIME 2.1. Ad hoc On-Demand Distance Vector (AODV) Routing is implemented in TRUE TIME 2.1. Here a comparison is made between the three Artificial Neural Network Architectures such as Feed forward neural network, Cascade forward neural network and Layered Recurrent Neural Network for various training functions like Levenberg-Marquardt back propagation (trainlm), Bayesian regularization back propagation (trainbr) and BFGS quasi-Newton back propagation (trainbfg) for Feed Forward Neural Network.
\end{abstract}

\section{Keywords}

AODV, Artificial Neural network, Cascade forward neural network, feed forward neural network, Layered Recurrent neural Network, Routing and Zigbee.

\section{INTRODUCTION}

A wireless Ad Hoc network is a distributed type of wireless network with no central controlling node. The network is called ad hoc because it does not rely on a pre-existing infrastructure, all nodes have equal status on a network and the nodes are permitted to associate with any other ad hoc network node in the link range. Wireless Ad-Hoc networks like Wireless Personal Area Network (WPAN) plays a very important role in today's industrial scenario. ZigBee is the best example of WPAN. ZigBee has been developed by the organization named 'ZigBee Alliance' [1] as a new wireless standard for the wireless solutions based upon the IEEE 802.15.4 [2] standard that provides low data rates, excellent performance in environments with low signal-to-noise ratio, less complexity, secure data transfer and provide batteries that can stay alive for longer times. The targeted applications of ZigBee are wireless sensors networks, interactive toys, home automation and remote controls.

Routing protocols has an important role in establishing and adopting the best path between source and destination nodes to minimize the time required to converge after the topology changes so that the data can be reached to the destination safely. Among various routing protocols available for ZigBee network like Cluster-Tree algorithm [1], [3], Ad hoc On Demand Distance Vector (AODV) routing algorithm [4-5] is generally used in ZigBee network to reduce cost and power consumption and improved reliability. There are various aspects or issues of network based on ZigBee wireless technology which need to be considered. In [6] authors have presented an energy-aware routing mechanism EA-AODV to maximize the use of the limited energy, also discussed the way of prolonging the lifetime of ZigBee network. Simulation result shows that the used energy routing mechanism is well suited for decreasing the energy consumption and can result into performance improvement of ZigBee network. Authors in [7] have emphasized on the critical problems of AODV like high data packet collisions, loss of data, and its inherent energy overheads makes AODV inappropriate for application which require effective, fast and consistent communications. Authors have proposed a fuzzy logic-based metric to be used in the decision making process of AODV. Experiment with new method AODV-FL shows better improvement in parameter like communication delay and number of packet loss leading to improved route efficiency. In [8] authors have proposed a new adaptive routing protocol for MANET called AODVLRT (AODV with Local Repair Trials) which reduces the routing messages the local repair algorithm used in the route maintenance of overhead resulted from the original AODV local repair algorithm. Proposed algorithm decreases both routing message overhead and the average end to end delay by on average $27.9 \%, 13.7 \%$ respectively less than the well-known AODV routing protocol which leads to higher throughput as compare to normal AODV routing protocol. [9] Proposes energy balance algorithm E-AOMDVjr to reduce the possibilities of loss of data transmission due to dying of low energy nodes in the network. Simulation shows that the improved algorithm improves the consistency of the network transmission, decreases the energy consumption and increases the lifetime of the network. [10] Proposes new routing protocol called ZFA based on flooding and AODV. This proposed protocol has higher reliability and less overheads as compare to AODV. [11] Aims at the problem of ZigBee AODVjr routing algorithm. Proposed algorithm simulation result indicates efficiently reduced energy power consumption and increased ZigBee network lifetime over traditional AODV.

Soft computing techniques viz. Fuzzy Logic, Artificial Neural Network, Artificial Neuro Fuzzy Inference System, Genetic Algorithm is basically introduced now days to optimize the performance of the system. In this paper we have proposed soft computing technique called Artificial Neural Network (ANN) for the optimization of transmission range of ZigBee network using AODV routing protocol in TRUE TIME/MATLAB. Proposed Algorithm improves the signal transmission range for a given data set containing the transmitted signal power and the threshold value of the received signal strength as input. This paper is organized as follows. Section 2, covers the necessary steps required for the simulation of AODV protocol using ZigBee network which requires the understanding of AODV flow and the TRUE TIME features. Section 3 describes the implementation of AODV simulator in TRUE TIME and its optimization using artificial neural network. 


\section{DEVELOPMENT FOR ZIGBEE NETWORK SIMULATION}

AODV [4-5] is the modest and broadly used algorithm for wired and the wireless network. It is one of the most efficient routing protocols in terms of establishing the shortest path between the source node and the destination node and using the lowest power consumption. It mainly uses the concepts of path discovery and maintenance. A path is established between the nodes only when there is a need to transfer data between nodes. It uses short messages called "HELLO" for the local connectivity. "HELLO" message is broadcasted by the source node to all the nearby nodes which is further flooded till it reaches to the destination node.

AODV mainly defines three types of messages: Route Requests (RREQs), Route Replies (RREPs) and Route Errors (RERRs) which is well explained in [4-5].

True Time is a Matlab/Simulink-based simulator, which provides co-simulation of controller task execution in realtime kernels, continuous plant dynamics and network transmissions [12-13].The simulator is presented in [Henriksson et al., 2003; Cervin et al. 2003; Henriksson et al., 2002; Andersson et al., 2005. This paper mainly focuses on the ZigBee protocol for the data transmission. ZigBee protocol mainly uses CSMA/CA [14] MAC protocol for the data transmission. For the simulation of AODV protocol for
ZigBee network certain parameters need to be adjusted as in [12] for each node in the network.

For the simulation, existing AODV model in MATLAB/TRUE [12] is used, as shown in figure 1, where the number of nodes has been increased to ten.

In order to handle the transmission and reception of data process in AODV routing protocol, two tasks are created in each node called AODVsend and AODVrecieve respectively. The AODV send task is activated from the application code as a data message should be sent to another node in the network. The AODV receive task handles incoming AODV control messages and forwarding of data messages. Communication between the application layer and the AODV layer is handled using TRUETIME mailboxes [12].

Other functional parameters are set as per requirement listed in Table 1.

The inputs for this AODV model are transmitting power and the threshold power and the output is transmission range. Simulation has been done for finding the transmission range for different set of transmitting power and the threshold power of range $[-1$ to $-10 \mathrm{dBm}]$ and $[-48 \mathrm{dBm}]$ respectively where Powers are converted in milliwatts and the range is in meters. This created set is used as input to the ANN network, which is explained in next section of this paper.
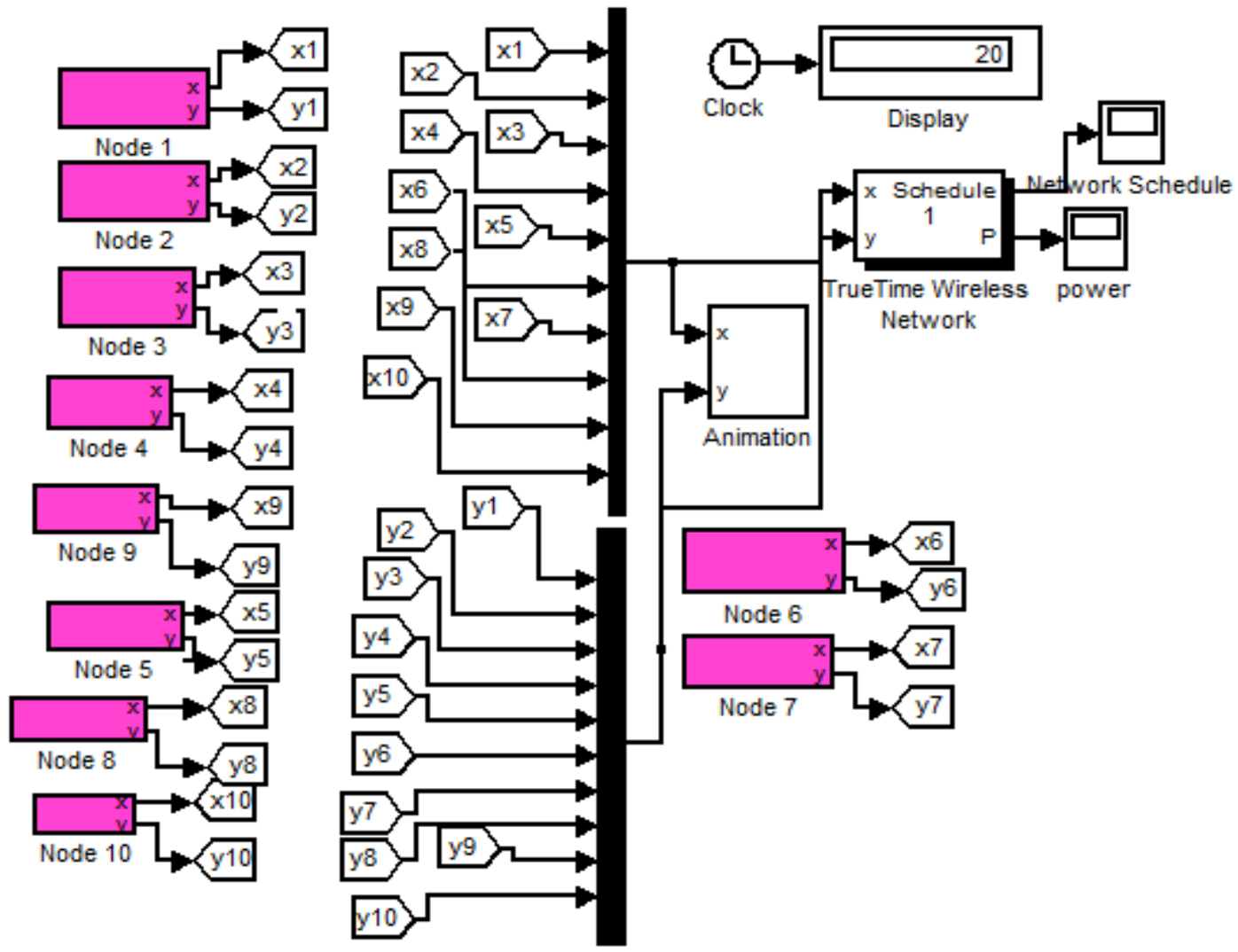

Fig 1: Modified AODV model with ten nodes 
Table 1: Parameter setup for AODV simulation

\begin{tabular}{|l|l|}
\hline Parameters & Description \\
\hline Network type & 802.15 .4 (ZigBee) \\
\hline No. of nodes & 10 \\
\hline Data rate (bits/seconds) & $250 \mathrm{kbps}$ \\
\hline No. of packets send & 40 \\
\hline Transmitting power (dBm) & -10 to 0 \\
\hline Threshold power (dBm) & -48 \\
\hline Minimum frame size (bits) & 248 \\
\hline ACK timeout (seconds) & 0.00004 \\
\hline Retry Transmission limit & 5 \\
& \\
\hline
\end{tabular}

\section{EVALUATION OF TRANSMISSION RANGE IN ZIGBEE NETWORK}

\subsection{Artificial Neural Network (ANN)}

An ANN is an association of simple processing elements called nodes which are linked together according to a specific network architecture. These nodes are inspired by neurons in the biological system. The objective of the neural network is to transform the inputs into significant outputs. Neural network is widely used in various field problems like clustering [20-23] and curve fitting [24]. Here feed-forward ANN (FFANN) has been employed, where there exist hidden layers between input and output layers [20]. It has an ability to represent almost all input-output relationships with a finite number of discontinuities as long as an appropriate size is assigned to the hidden layer [20], [23]. Figure 2 shows that in feed forward neural network there does not exist any feedback between the input and the output. Shown figure is created using NNTOOL in MATLAB.

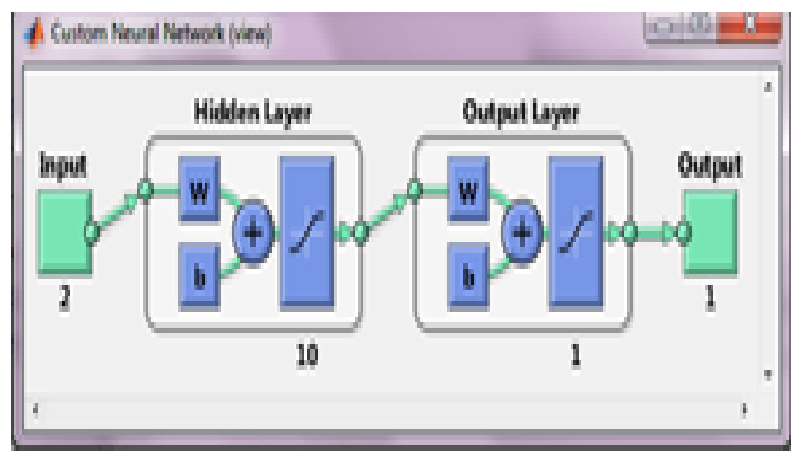

Fig 2. Generated Artificial Neural Network

It can be seen that each layer either it is a hidden layer or an output layer, it contains a weight matrix, a bias vector, a sum operator, a transfer function (TF) and an output vector. Each input is weighted with an appropriate weight $w$. The summation of the weighted inputs and the bias forms the input to the transfer function block. The input-output relationship in either layer can be represented by [20]

$a=f\left(W^{T} p+b\right)$

Where $p$ is the input vector, $W$ is a weight matrix, $b$ is a bias vector, $f$ is a transfer function (TF) and $a$ is an output vector. Other important information regarding theoretical background and working mechanism of artificial neural network (ANN) can be referred from [22] and [23-25].

Table 2: Simulation Parameters configuration

\begin{tabular}{|c|c|}
\hline $\begin{array}{c}\text { Network } \\
\text { parameters }\end{array}$ & features \\
\hline Network type & Feed-forward neural network \\
(FFNN)
\end{tabular}

Here, a comparative study has been done between the three training function or algorithms like Levenberg-Marquardt back propagation (trainlm), Bayesian regularization back propagation (trainbr) and BFGS quasi-Newton back propagation (trainbfg) for Feed Forward Neural Network. Network parameters set for the creating network for Feedforward neural network, cascaded neural network and the layer recurrent neural network are listed in table 2. Details regarding these neural network can be obtained from [26-27].

The data taken for ANN is the data set created using the simulation of AODV model in TRUE TIME. Variables Tx_pow and the Thres_pow are the inputs associated with the transmitting power and the threshold power respectively in AODV Simulink model whereas output Range is the transmission range taken as output of AODV model. The overall process is shown in figure 3 . 
TRUETIME/MATLAB
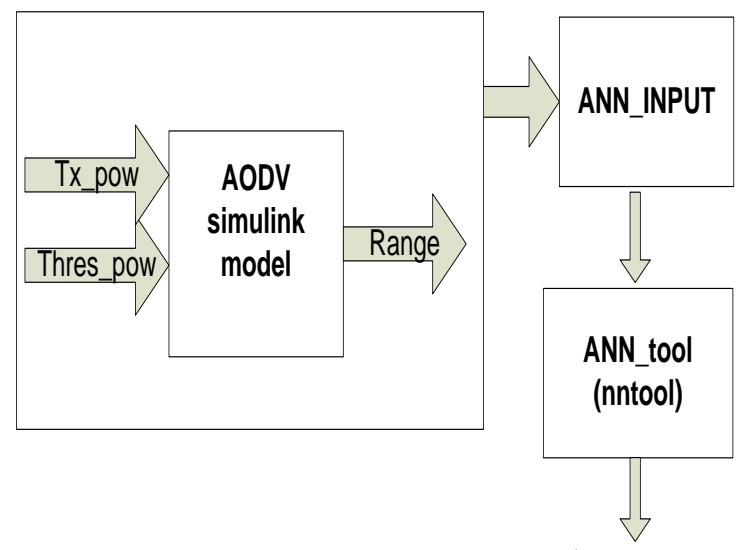

ANN_output

\section{RESULTS AND CONCLUSION}

A comparative study has been done between the various Back propagation training algorithms like Bayesian regularization back propagation (trainbr), BFGS quasi-Newton back propagation (trainbfg) and Levenberg Marquardt back propagation (trainlm) in ANN as shown in figure 4-6. It has been observed that the trainbr perform comparatively better as compare to other two training methods for finding the transmission range for the Zigbee network using AODV routing protocol. In figure 4 to 6 it can be seen that the Layered Recurrent Neural Network gives higher transmission range as compare to the traditional method based on the AODV simulator in TRUE TIME 2.1 in MATLAB.

Fig 3. Overall ANN simulation process

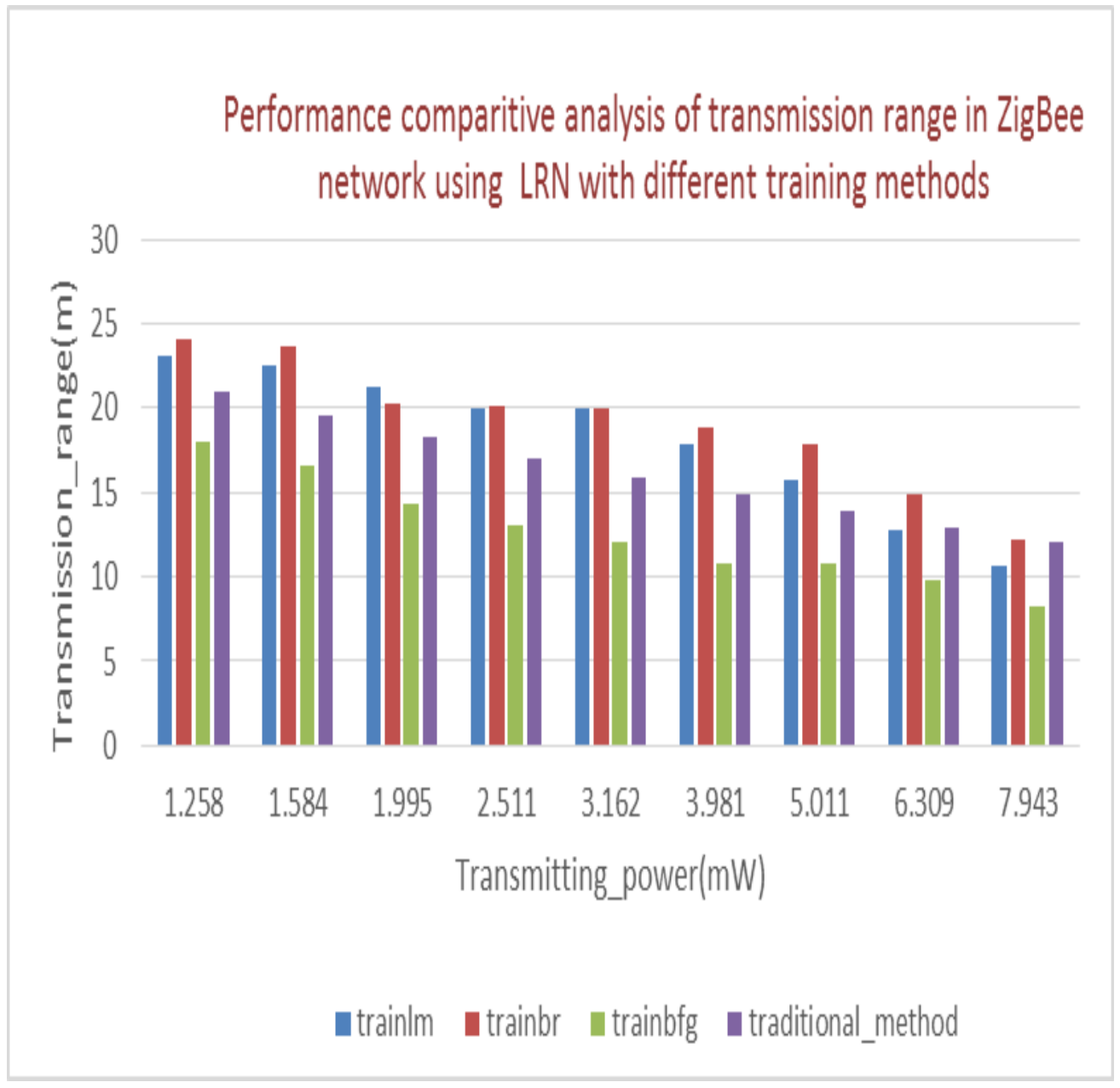

Fig 4: comparison of various training algorithms for layer recurrent network in ANN 


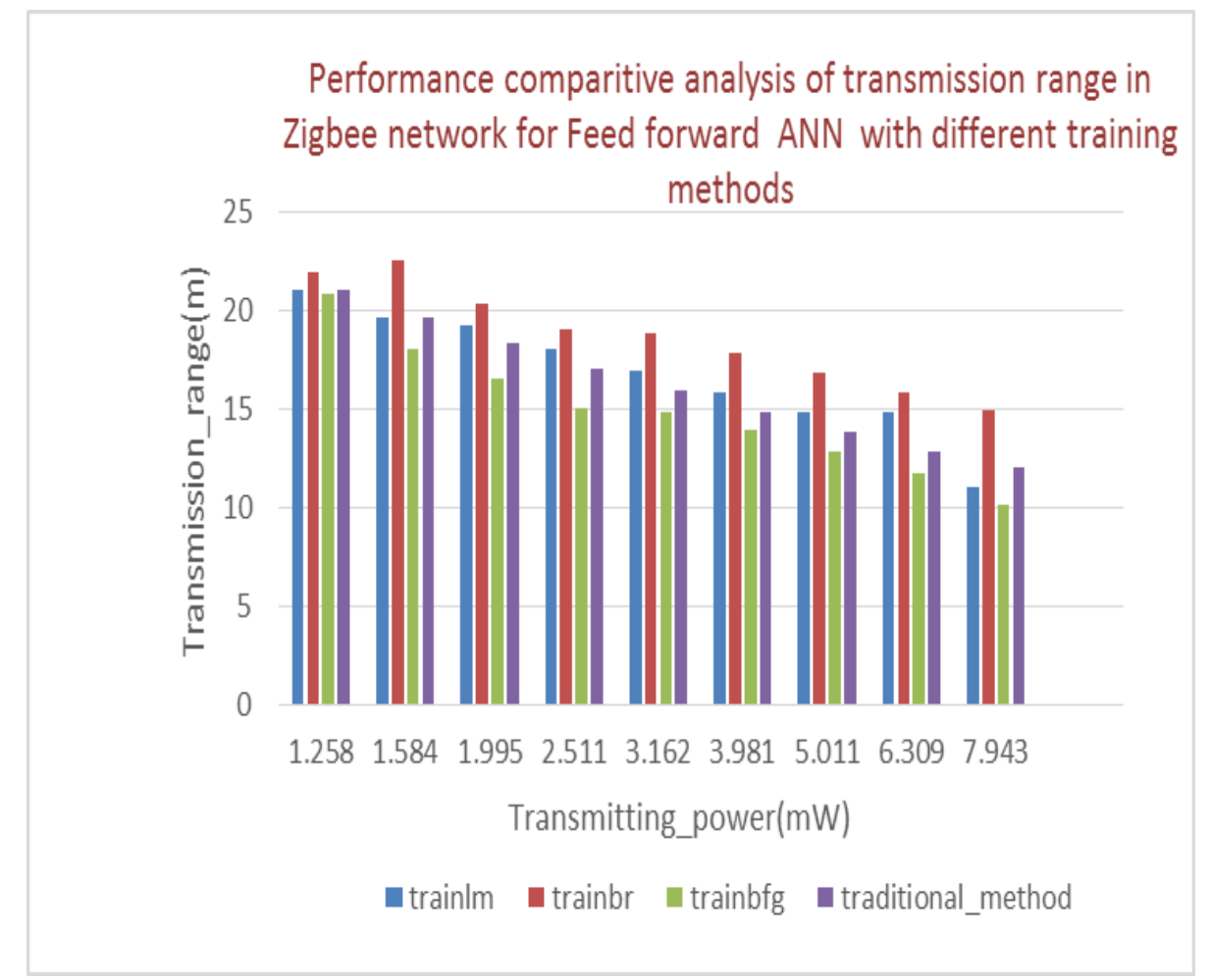

Fig 5: comparison of various training algorithms for feed forward network in ANN

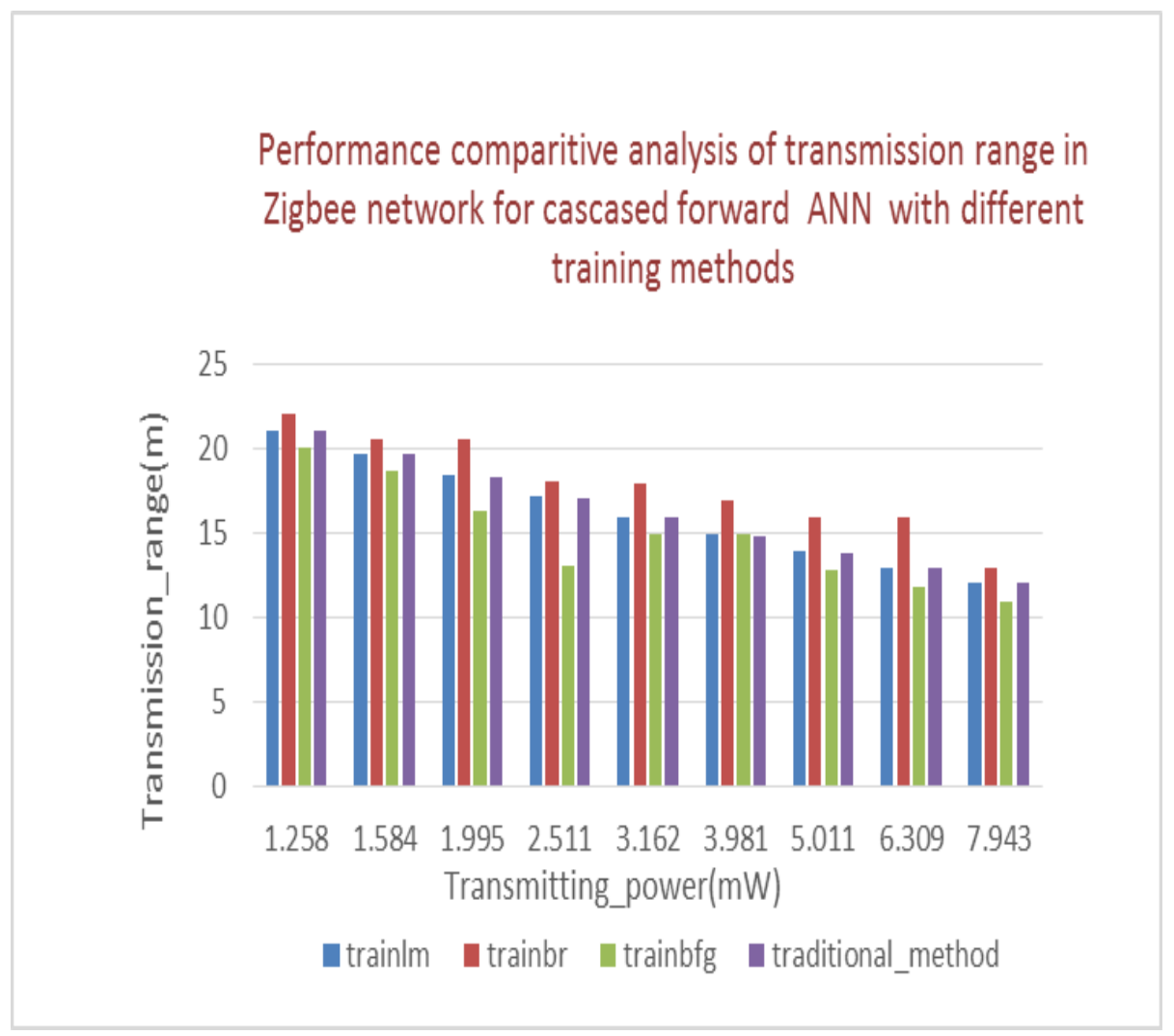

Fig 6: comparison of various training algorithms for cascaded feed forward network in ANN

\section{ACKNOWLEDGMENTS}

I am thankful to my husband Mr. Pranav Saraswala and Associate Professor Mrs. Anuradha Deshpande, Electrical Engg Department, The Maharaja Sayajirao University of Baroda for their generous upkeep and stimulus for writing this paper.

\section{REFERENCES}

[1] ZigBee Alliance, Network Layer Specification 1.0, Dec. 2004,[Online].Available:http://standards.ieee.org/getieee 802/download/802.15.4-2003.pdf 
[2] Institute of Electrical and Electronic Engineers, Inc., "IEEE Std. 802.15.4-2003, IEEE Standard for Information Technology, Telecommunications and Information Exchange between Systems - Local and Metropolitan Area Networks - specific Requirements Part 15.4 : Wireless Medium Access Control (MAC) and Physical Layer (PHY) Specifications for Low Rate Wireless Personal Area Networks (LR-WPAN).

[3] Ran Peng, Sun Mao-heng, Zou You-min, "ZigBee Routing Selection Strategy Based on Data Services and Energy-balanced ZigBee routing", Proceedings of the IEEE Asia-Pacific Conference on Services Computing, 12-15 Dec. 2006

[4] C. Perkins and E. Royer, "Ad Hoc On-demand Distance Vector (AODV) Routing" , Internet Draft, MANET working group, draft-ietf-manetaodv- 05.txt, March 2000 .

[5] C. E. Perkins, E. M. Royer, and S. R. Das, "Ad hoc on demand distance vector (AODV) routing," IETF Internet draft, draft-ietf-manetaodv- 13.txt, Feb. 2003.

[6] YiGong Peng, YingLi Li, ZhongCheng Lu, JinShou Yu, "Method for Saving Energy in Zigbee Network", 5th International conference on Wireless Communications, Networking and Mobile Computing, 24-26 Sept. 2009.

[7] Antonio M. Ortiz, Fernando Royo, Teresa Olivares and Luis Orozco-Barbosa, "Intelligent Route Discovery for ZigBee Mesh Networks ", IEEE International Symposium on World of Wireless, Mobile and Multimedia Networks, 20-24 June 2011.

[8] Maged Salah EldinSoliman, SherineMohamed Abd Elkader, Hussein SherifEissa, HodaAnis Baraka, "NEW ADAPTIVE ROUTING PROTOCOL FOR MANET", published in Ubiquitous Computing and Communication Journal Volume 2,Number 3, pp-16-22.

[9] Jun Xiao and Xiaojun Liu, "The Research of EAOMDVjr Routing Algorithm in ZigBee Network", IEEE conference on Control and Decision Conference (CCDC), pp. 2360 - 2365 23-25 May 2011.

[10] Zheng Sun, Xiao-guang Zhang, Dianxu Ruan, Hui Li and Xun Pang, "A Routing Protocol based on Flooding and AODV in the ZigBee Network", International Workshop on Intelligent Systems and Applications, pp. 1 - 4 23-24 May 2009.

[11] Zhao Hong-tu , Ma Yue-qi , "Improved Routing Algorithm Research for ZigBee Network ", Proceedings of the Third International Symposium on Computer Science and Computational Technology, pp. 017-020, Jiaozuo, P. R. China, 14-15,August 2010

[12] M. Ohlin, D. Henriksson, and A. Cervin, "TrueTime 2.1 Reference Manual," Department of Automatic Control, Lund University, Sweden, 2007. [Online] Available: http://www.control.lth.se/truetime.

[13] Andersson, Martin, Dan Henriksson, Anton Cervin and Karl-Erik arzen (2005). "Simulation of wireless networked control systems", Proceedings of the 44th IEEE Conference on Decision and Control and European Control Conference, Seville, Spain, 2005

[14] Tamer M.S. Khattab, Mahmoud T. El-Hadidi and HebatAllah M. Mourad, "Analysis of Wireless CSMA/CA Network Using Single Station Superposition (SSS)", published in International Journal of Electronics and Communications, Vol. 56, pp. 71-81, 2002

[15] Pejman Tahmasebi, Ardeshir Hezarkhani, “Application of Adaptive Neuro-Fuzzy Inference System for Grade Estimation; Case Study, Sarcheshmeh Porphyry Copper Deposit, Kerman, Iran", published in Australian Journal of Basic and Applied Sciences, ISSN 1991-8178, pp. 408-420, 2010

[16] Yevgeniy, Gershteyn Larisa Perman, "Matlab: ANFIS Toolbox", presented on 04/22/2003

[17] Jyh-Shing Roger Jang, "ANFIS : Adaptive-NetworkBased Fuzzy Inference System ”, IEEE transactions on systems, man and cybernetics, vol. 23, may-june 1993

[18] Dr. Bob John, “ANFISnote” [Online]. Available www.scribd.com/doc/134927592/ANFISnote

[19] The Fuzzy Logic Toolbox for use with MATLAB, J.S.R. Jang and N. Gulley, Natick, MA: The Math Works Inc., 1995

[20] Yizheng $\mathrm{Xu}$, Graduate Student Member, IEEE, and Jovica V. Milanovic, Fellow, IEEE, "ArtificialIntelligence-Based Methodology for Load Disaggregation at Bulk Supply Point", published in IEEE transactions on power systems, Vol. 30, MARCH 2015

[21] G. Chicco, R. Napoli, and F. Piglione, "Comparisons among clustering techniques for electricity customer classification," published in IEEE transaction. Power Syst., vol. 21, pp. 933-940, May 2006.

[22] L. Du, D. HE, Y. Yang, J. A. Restrepo, B. Lu, R. G. Harley, and T. G. Habetler, "Self-organizing classification and identification of miscellaneous electric loads," in Proc. IEEE PES Gen. Meeting, San Diego, CA, USA, pp. 1-6, 2012.

[23] Mark Hudson Beale, Martin T. Hagan, Howard B. Demuth, "Neural Network Toolbox ${ }^{\text {TM }} 7$ User's Guide", 2009

[24] CIGRE Working Group C4.605, Rep.5, "Modelling and Aggregation of Loads in Flexible Power Networks," (566), Feb. 2014

[25] H. Ku-Long, Y.-Y. Hsu, and Y. Chien-Chuen, "Short term load forecasting using a multilayer neural network with an adaptive learning algorithm," IEEE Trans. Power Syst., vol. 7, no. 1, pp. 141-149, Feb. 1992.

[26] Saduf, Mohd Arif Wani, "Comparative Study of Back Propagation Learning Algorithms for Neural Networks", pp. 1151-1156, Volume 3, Issue 12, December 2013.

[27] M. Riedmiller, H. Braun, "A direct adaptive method for faster back-propagation learning: the RPROP algorithm, ", Proc. of the International Conference on Neural Networks, pp. 586-591, 1993 